\title{
El sentido de cuidar a la familia, desde la perspectiva de Bourdieu
}

Mtra. Rocío del Carmen Guillén Velasco*, Mtra. Laura Morán Peña**

*Maestra en Enfermería con Énfasis en Salud Comunitaria. Profesor Asociado B ENEO-UNAM. México, **Maestra en Educación Superior y candidata a Doctora en Ciencias de la Educación. Profesor Titular ENEO-UNAM. México.

Este artículo es derivado del trabajo de investigación El cuidado a la familia: ¿qué significa para las Enfermeras comunitarias? que para obtener el grado de Maestría en Enfermería se realizó en el 2003. Es un estudio de corte cualitativo que rescata el sentido que las Enfermeras comunitarias le otorgan al cuidado con enfoque familiar. Participaron 4 Enfermeras comunitarias del Estado de México, con un perfil muy homogéneo, seleccionadas mediante muestreo teórico. Para la recolección de información se utilizó la entrevista semiestructurada y el análisis de la información se realizó a través de codificación abierta, axial y selectiva.

La interpretación de los hallazgos se hizo alrededor de los conceptos de campo, habitus y capital cultural que el mismo gremio de Enfermería impone al ingresar al campo laboral. Esto permitió el anclaje teórico, confirmando y comprendiendo lo que informaron las Enfermeras. En la realidad social, la práctica del cuidado queda integrada, como una consideración global al momento de ofrecer la interacción Enfermera-persona cuidada. Las Enfermeras en su práctica, manifiestan una paradoja entre lo que es trabajo profesional de Enfermería y el deber ser, pues el campo, el habitus y los capitales que se distribuyen en dicha práctica, están en lógicas diferentes y por tanto estratificadas y desniveladas.

Como conclusión, el cuidado y la familia demandan la reflexión disciplinar, que reconozca la necesidad del carácter científico constitutivo de los mismos, la explicación de los fenómenos relacionados y mayor deliberación acerca de los códigos de lenguaje con el que se construyen dichas explicaciones.

PALABRAS CLAVE: Cuidado, Enfermera comunitaria, campo, habitus, capital cultural, valores, práctica del cuidado, familia.

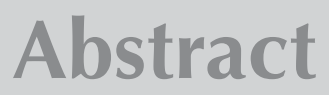

This article is derived from the investigation The family's care: what means for the communitarian nurses? that to choose for degree of Mastery in Nursing it was realized in 2003. This qualitative-type study, rescues the sense communitarian nurses give to care with familiar approach. It was performed with four communitarian nurses from the state of Mexico participated, who have a very homogeneous profile and were selected through theoretical sampling. For data collection was used a semi-structured interview, and open axial and selecting coding performed data analysis.

Finding was interpreted around cultural field, habitus and capital that nurses guild itself imposes when entering the laboral market. This allowed the theoretical anchoring, assuring and understanding what nurses informed. Within social reality, care practice gets integrated as a global consideration at the moment when the cared person-nurse interaction is offered. Nurses manifest a paradox between what the social nurses doing are and what it must be, because, the field, habitat and capitals distributed are within different logics, and therefore, they are stratified and uneven.

Concluding, care and family, demand disciplinary reflection that acknowledges the need for the scientific nature that constitutes it, the explanation of phenomena related to care, and mayor reflection about the language codices with which such explanation of the phenomena related to care are constructed.

KEY WORDS: Care, community nurse, field, habitus, capital, values, practice of the care, family. 


\section{INTRODUCCIÓN}

El artículo describe una aproximación al sentido que las Enfermeras comunitarias le otorgan al cuidado que proporcionan a la familia desde la perspectiva de Bourdieu', entendido como todas aquellas acciones y conocimientos incrustados en una serie de prácticas que llevan a cabo las Enfermeras comunitarias y que muchas veces son compartidas por la propia comunidad profesional.

Es así, que el cuidado interpretado desde diversos campos atiende a condiciones de producción distintas y tendrá sentidos diferentes acorde a experiencias, a conocimientos, a circunstancias sociales de cada Enfermera. Asumir al cuidado profesional de Enfermería como un proceso socialmente construido supone, también, mirar a la sociedad en general como un proceso dinámico en el que se realizan acciones, se producen discursos y se da sentido al mundo a partir de complejos procesos sociales e intelectuales, siempre desde un lugar situado e históricamente construido.

Las Enfermeras, según la posición que ocupen por su nivel laboral, por su formación académica o por su afiliación con modelos disciplinarios, negociarán con y a partir de esas condiciones sociales para elaborar un relato subjetivo sobre el cuidado que no será de modo necesario coherente y automáticamente transparente, pero en cuya expresión pueden encontrarse las huellas de sus anclajes de identidad o de su relación (por afirmación o negatividad) con ciertos campos discursivos y de su pertenencia a una formación específica.

Dentro del contexto del cuidado se consideran cuatro aspectos que parecen ser trascendentales para comprender la parte intangible del cuidado y que tienen que ver con la forma en que se movilizan las Enfermeras en la práctica: a) el cuidado aún estando presente en el discurso de Enfermería, no es considerado en los escenarios de práctica como elemento central y fundamental del proceso de Enfermería; b) el cuidado en todas sus formas ha sido el componente de ayuda profesional más ignorado; c) con la creciente tecnologización, la tendencia en el cuidado de Enfermería es la disolvencia de su práctica ancestral como elemento central y d) las Enfermeras construyen esta noción a partir de reflexionar cómo el cuidado subyace en su práctica cotidiana, lo que para ellas y sus usuarios significa interactuar en ese proceso interpersonal en el momento en que se hace conciencia de él.

De manera tradicional las prácticas de Enfermería se llevan a cabo en servicios hospitalarios, con un fuerte énfasis técnico así como en la formación profesional siendo ésta una práctica hegemónica, sin embargo, en el desarrollo disciplinario y en la creciente necesidad profesional de

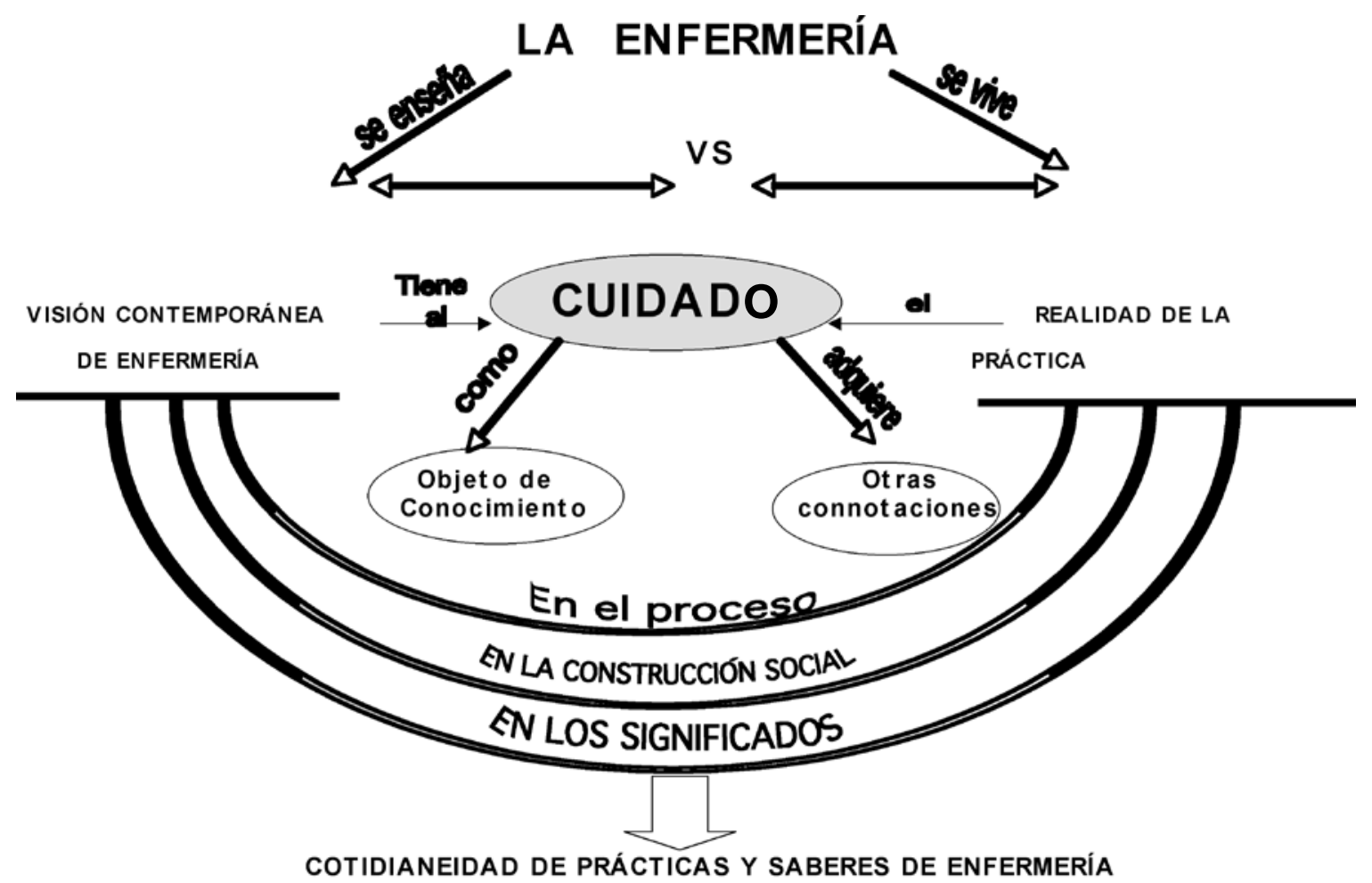

Figura 1. Mapa conceptual construido para la investigación 
definir el campo de Enfermería, el cuidado de Enfermería empieza a formar parte del discurso.

En los programas de estudio actuales para la formación de Enfermeras, con énfasis en los componentes técnico y tecnológico, se considera que no solamente la parte del hacer es importante, sino la del desarrollo del ser, tanto de la Enfermera como de la persona cuidada que se trasforman en el momento de la interacción. Los programas de estudio pretenden ayudar al futuro profesional a descubrir y asimilar valores universalmente reconocidos como deseables y buscan las estrategias para ayudarlos en el desarrollo y formación de sus capacidades que orienten su juicio ante situaciones que plantean un conflicto de valores, la formación de actitudes y aplicación de normas de convivencia que les permitan alcanzar su propio proyecto de vida, en el que, de muchas maneras permea el cuidado como elemento central de trabajo de Enfermería.

Sin embargo, es muy interesante cuando hay un cambio de racionalidad en la forma en que tradicionalmente se ubica al cuidado, especialmente el dirigido al grupo familiar dentro de la práctica comunitaria. La realidad es que lo que se enseña y se aprende en la escuela tiene poca pertinencia en la práctica real, pues ocurren en lógicas, en momentos y en contextos diferentes ${ }^{2}$ (figura 1).

Lo que se observa es que éste se aplica de una manera muy intuitiva y no sistemática y, hay entonces, durante la práctica, rupturas en la forma de actuación, por lo que surgen dos inquietudes fundamentales en la investigación: ¿qué es el cuidado? y ipor qué trabajar específicamente con familia?

Ante tales cuestionamientos, es importante esclarecer por qué estas dos situaciones son básicas para el trabajo enfermero comunitario y entender como se construyen estas situaciones en el escenario de práctica comunitaria para tratar de definir un marco conceptual sobre cuidados comunitarios profesionales de Enfermería.

Lo interesante del estudio es que las Enfermeras manifiestan que sus actividades tienen que ver con el concepto cuidado descrito ya en múltiples trabajos y el trabajar con familias les significa realmente esa capacidad de desarrollar "ayuda a otros", "ser confidentes", "ponerse en el lugar de los otros", "escuchar, "servir", con lo cual, se reconoce una mirada diferente de lo que significa el cuidado meramente pues tiene más que ver con la interacción humana que con las técnicas y procedimientos que tanto se ha privilegiado en la práctica y en la formación. De esta manera, las actividades que realizan las Enfermeras comunitarias se pueden considerar como aquellas actividades cuidativas que realizan donde cuidar es un fenómeno emocional que involucra los sentimientos de amor y afecto y de apoyo emocional, pero que enfrentadas a la realidad laboral, pocas veces se reconocen pues están inmersas en una realidad social que las engloba y dirige su accionar.

\section{METOdOLOGÍA Y PROCEDIMIENTO}

La investigación se desarrolló con base en una metodología cualitativa. ${ }^{3-9}$ Se determinó trabajar con Enfermeras comunitarias; participaron 4 informantes, con un perfil muy homogéneo: todas mujeres, con promedio de edad de 39.5 años, residentes en el Estado de México, casadas, madres de familia. Trabajadoras en unidades de primer nivel de atención con experiencia promedio de 20 años. La muestra de informantes fue seleccionada utilizando la técnica de muestreo teórico. ${ }^{10-13}$

Para la recolección de información se utilizó la entrevista con preguntas abiertas siguiendo un orden mediante una guía. ${ }^{14}$ Para registrarlos se utilizó audiograbación y se consideró una sola entrevista.

Con el objeto de hacer la codificación y análisis, se procedió a la trascripción de las entrevistas tal y como se escuchan en la cinta de audio. Posteriormente, se llevó a cabo una primera lectura general a las entrevistas ya transcritas; se realizaron lecturas consecutivas de cada entrevista ${ }^{15,16}$ y por cada párrafo lo que permitió la identificación y clasificación de elementos, la separación de las unidades y la síntesis y agrupamiento, para hacer aproximaciones como categorías a través de procesos de reducción inductiva. ${ }^{17,18}$

El análisis de la información fue realizado siguiendo tres procedimientos de codificación: abierta, axial y selectiva. ${ }^{19}$ Así mismo, el análisis de contenido se utilizó para estudiar la información. ${ }^{20-22}$ Para la elaboración de las conclusiones se seleccionó a la teoría anclada o grounded theory, ${ }^{23-25}$ porque se trató de entender la experiencia cotidiana tal como el actor social para obtener conocimientos de ella. Esto se logró a partir de los textos recolectados en contextos naturales y sus hallazgos son formulaciones teóricas de la realidad que permitieron la consolidación teórica y aplicación de otras teorías para establecer relaciones y conexiones importantes en los hallazgos.

Para llegar a la comprensión del fenómeno, las categorías de análisis se ordenaron en tres conceptos específicos: campo, habitus y capital. Considerando los conceptos anteriores, se abrió un gran abanico de posibilidades para 
poder comprender el mundo del cuidado a la familia donde se mueven las Enfermeras comunitarias, pues estos conceptos son sistemáticos y pueden trasponerse de un ámbito de práctica a otro, de un campo a otro, lo que permite presentir, en cierta manera, como va a actuar la Enfermera en una situación determinada, después de haberla visto actuar en situaciones previas.

Con el objeto de estimar la validez de los resultados se regresó con las Enfermeras que proporcionaron la información, se trianguló con la literatura y con otras profesionales expertas en la Enfermería. ${ }^{26,27}$

Para comprender el cuidado a la familia, la información se interpretó y organizó alrededor de dos grandes temas: las representaciones de las Enfermeras comunitarias acerca del cuidado y las actividades cotidianas de las Enfermeras con la familia en el trabajo comunitario estructurando la matriza axial (figura 2). Partiendo de esta matriz axial, se construyeron las categorías selectivas: 4 categorías sobre el cuidado y 3 categorías sobre el trabajo con familia en la comunidad. Posteriormente se elaboró la matriz temática

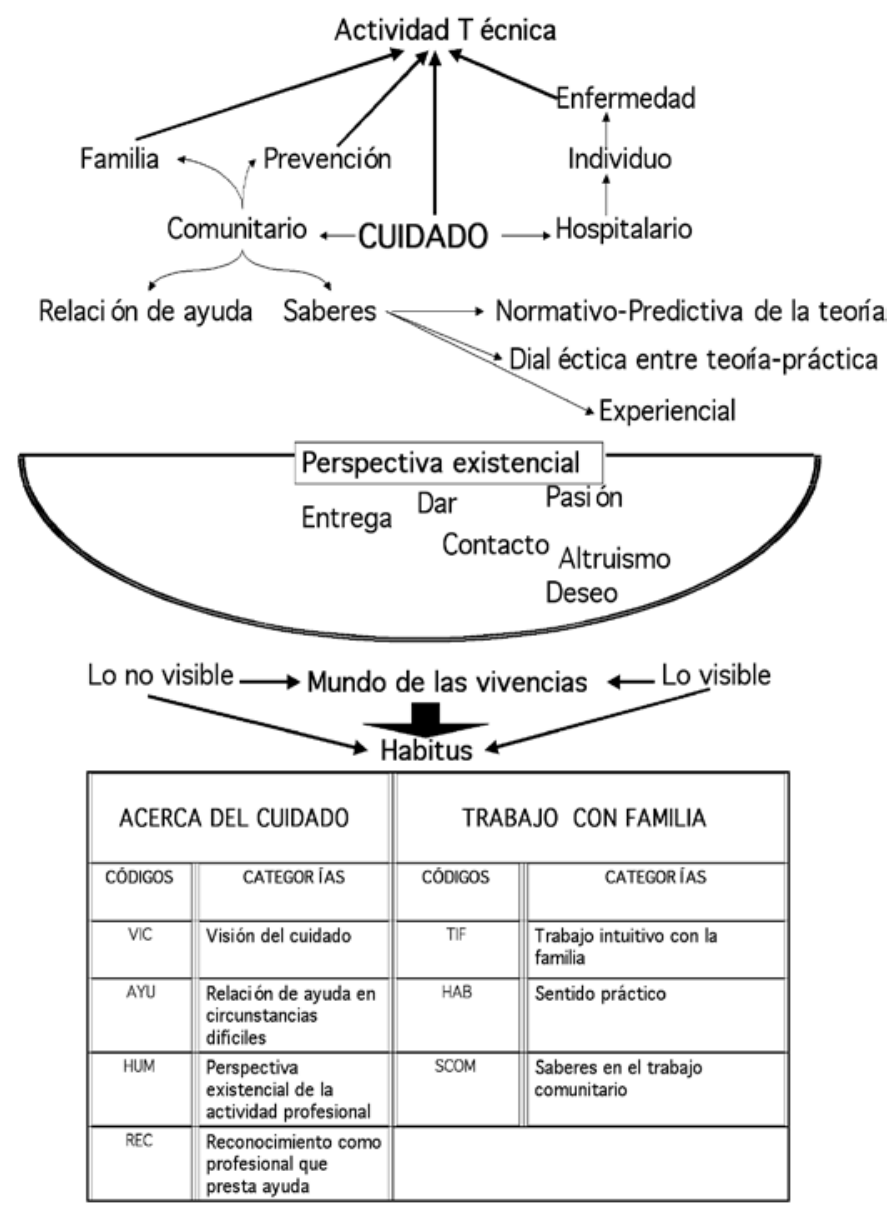

para reestructurarlas en 3 grandes dimensiones o ejes que pueden servir como orientadores para la descripción del significado que este fenómeno tiene (figura 3).

\section{DISCUSIÓN}

Para comprender el sentido del cuidado dentro del trabajo profesional de las informantes, se optó por los conceptos de campo, habitus y capital, ${ }^{28-33}$ con la esperanza de que afloren por proyección o resonancia las categorías que se estructuraron para este trabajo.

Desde anclajes particularmente sociológicos, emergió lo significativo de las relaciones sociales; desde lo extremadamente simple y obvio hasta lo complejo y oculto, marcado siempre por una expresión cultural que escapa a hacer generalizaciones. El capital, el habitus y el campo específico de Enfermería en la comunidad aparecen a partir de una práctica específica, de su historia, de las experiencias que cada una de las informantes les asigna y resignifica a partir de su relación con el pasado, presente y futuro. Aunque también, las estructuras de poder, el papel de los significados rituales, la construcción social, se interrelacionan finalmente, a partir de un medio ambiente y del mismo entorno social, y todo esto explica la gran complejidad de lo social.

Por otro lado, la relación de fuerzas resultantes de la desigual distribución del capital o de los capitales que están en juego en la práctica comunitaria, es los que definen las posiciones dominantes y dominadas dentro de este campo y, por tanto, la capacidad de ejercer un poder e influencia sobre los otros. El hecho es que las Enfermeras y todos los elementos con los que ejercen fuerza social, disponen de bienes económicos y culturales que son fuentes de poder con respecto a los que los poseen en menor medida o simplemente carecen de ellos.

Encontrado en el discurso de las Enfermeras comunitarias lo anterior, se buscó una interpretación del cuidado y el trabajo con la familia a través de las siguientes dimensiones: filosófica-axiológica, sociológica-práctica y educativacognitiva.

Dimensión filosófica-axiológica del cuidado. Reconocen la parte invisible del cuidado a través de la relación de ayuda y sus componentes (transacciones de responsabilidad propia de la Enfermera, las cuales tienen que ver con su conciencia de ser y la del otro). Esto es importante pues se requiere hacer consciente lo que el cuidado significa dentro de la práctica profesional, pues es una forma de 
asumir y llevar a cabo un proyecto de vida. El "ponerse en lugar de otro", "escuchar", "servir" son conceptos que con mucha frecuencia utilizan las informantes. Por otra parte, el cómo entienden el enfoque familiar tiene que ver con sus propios esquemas referenciales de pertenencia a una familia. Atienden muchos aspectos de la relación de ayuda que sienten y saben de manera intuitiva que deben trabajar como son: "ayudar", "aconsejar", "orientar", "ser confidente".

Las informantes aún cuando son expertas realizando su trabajo, comparten una serie de sentimientos como el miedo, la dependencia, la vigilancia y la supervisión administrativa, que no les permite ser creativas y re-crear su escenario profesional, sin embargo, es muy interesante conocer que tienen un gran potencial para desarrollar su trabajo pues les encanta su trabajo, tienen gusto por hacerlo, interés en las personas, compromiso, orgullo, necesidad de saber más, dedicación, satisfacción, además de "ponerse la camiseta", "ser humanistas", "amar la carrera" "ver frutos de lo que hacen".

Dimensión sociológica-práctica del cuidado y del trabajo comunitario. La práctica de una profesión no es un mero hacer, sino que se construye mediante un sentido y un significado social, histórico y político, por tanto, intencional.
Es interesante cómo se movilizan las Enfermeras comunitarias dentro de la práctica social ya que son sujetos con una forma muy particular de ver al mundo y en consecuencias como actúan en él.

Así el campo en el que se mueven tiene que ver con las estructuras sociales externas como el sistema de salud vertical, trabajo con base a los programas emitidos de nivel central y con enfoque de riesgo, el cual privilegia las actividades fragmentadas, episódicas, muy normativas, con indefinición de tareas y actividades, ejercicio del poder de manera vertical y promoción de una actitud dependiente, además de una visión reduccionista del trabajo de Enfermería impuesta o autoimpuesta que produce un trabajo instrumental y una práctica dependiente, vislumbrado también como un trabajo doméstico y femenino. Es de notar, que en el discurso de las Enfermeras comunitarias, refieren un gran compromiso con sus actividades cotidianas; la motivación intrínseca es un elemento importante en su trabajo, así como el establecimiento de relaciones interpersonales con las personas que tienen a cargo. La mayoría de las actividades realizadas con la familia y con la propia comunidad se observan de control, de vigilancia, de supervisión, sanción, control del individuo, normalización, entendiéndose como situaciones punitivas. $^{34}$

\section{Figura 3. Matriz temática}

\begin{tabular}{|c|c|c|}
\hline DIMENSIÓN (EJE TEMÁTICO) & SIGNIFICADO DEL CUIDADO & $\begin{array}{c}\text { ACTIVIDADES COTIDIANAS CON LA } \\
\text { FAMILIA EN EL TRABAJO COMUNITARIO }\end{array}$ \\
\hline FILOSÓFICA - AXIOLÓGICA & $\begin{array}{c}\text { Visión del cuidado, relación de ayuda } \\
\text { en circunstancias difíciles, perspectiva } \\
\text { existencial de la actividad profesional }\end{array}$ & Sentido práctico \\
\hline SOCIOLÓGICA - PRÁCTICA & $\begin{array}{r}\text { Reconocimiento como profesional que } \\
\text { presta ayuda }\end{array}$ & Trabajo intuitivo con familia, saberes en el \\
trabajo comunitario
\end{tabular}


Por otra parte, el habitus tiene que ver con las formas sociales internalizadas en forma de esquemas de percepción, pensamiento y acción. A través de esto las informantes han sido moldeadas a operar de una forma establecida y asumida por la misma comunidad profesional.

El sistema de salud, el sistema educativo y las redes de Enfermería definen de manera intencionada o no, ciertas formas de pensamiento y de apreciación del mundo, las cuales generarán la práctica tal y como se observa. A través de esto las Enfermeras han sido inculcadas e incorporadas a operar de una forma establecida y asumida por la misma comunidad profesional. Esto supone que las Enfermeras no actúan libremente: sus prácticas están condicionas por toda la historia anterior y que ellas reproducen de una forma tal para que siga operando de esa manera.

Dimensión educativa-cognitiva del trabajo comunitario con la familia. La educación de Enfermeras tiene lugar en situaciones sociales de gran complejidad y ambigüedad, que se supone no pueden resolverse con la mera aplicación de conocimientos científicos. Se entiende que la Enfermería tiene un gran componente técnico pero hay que reconocer que se encuentra en franco desbalance respecto a los aspectos éticos y de conocimiento (obrar, saber y hacer).

Las informantes reconocen un abismo en su formación profesional pues la mayoría de las Enfermeras que trabajan en la comunidad son auxiliares de Enfermería con conocimientos incipientes de la disciplina y por otro lado, la solicitud de la práctica respecto a las competencias profesionales que les exige desarrollar como la atención directa a personas, familias, grupos de riesgo y comunidad a través de un sinnúmero de estrategias tales como: la gestión para la atención, la comunicación y negociación, valores y deontología, el manejo de recursos para la salud, el trabajo en equipo, el trabajo intersectorial para la salud, la investigación, la mejora continua de la calidad de la atención, las intervenciones basadas en la evidencia científica, la seguridad de los pacientes y la comunidad, la participación en las normativas y legislación para la salud, la asesoría a personas en materia de salud, la acción para la cooperación en salud internacional, y la investigación, la formación continuada y el desarrollo profesional.

Lo anterior se entiende pues ellas forman parte de un conglomerado profesional marginado de los avances de salud, de educación y de presupuesto. Además se percibe que el cuidado, como elemento central del discurso de Enfermería, no se enseña ni se aprende ni se adopta como una forma de vida. Lo que el curriculum de Enfermería ha privilegiado es el componente técnico medicalizado y deja de lado al ser humano y al cuidado.

Los saberes de las Enfermeras comunitarias se derivan de tres concepciones: normativo-predictiva de la teoría, dialéctica entre teoría-práctica y la experiencial35,36 de donde surgen obstáculos para trabajarlos en su cotidiano, esto es, problemas en su actuar en general, problemas vividos durante la formación y que les hacen "ruido" y otros muchos que se les presentan, a partir de su experiencia para solucionar los problemas que se les presentan durante su ejercicio laboral.

Se observan por tanto rupturas muy importantes entre lo que han aprendido y lo que el campo les pide en su actuar. Principalmente se observa que el cuidado como concepto no es enseñado ni aprendido en ningún espacio de formación. Con este desconocimiento de la importancia de los cuidados relacionados con el mantenimiento de la vida o de los habituales, las Enfermeras descuidan gravemente todo lo importante para un niño, una mujer, un adulto o un viejo en sus necesidades cotidianas. Haciéndose cada vez más curativos los cuidados que proporcionan, han perdido de vista y dejado de lado todo aquello que tiene sentido para garantizar la vida y su razón de ser, creando un abismo entre el cuidado curativo y el cuidado habitual ya que hablan exclusivamente de enfermedades, tratamientos invasivos y no invasivos, alta tecnología y no toman en cuenta todas aquellas prácticas que tienen que ver con los usos y costumbres habituales del individuo para conservar su salud.

\section{REFLEXIONES FINALES}

Desde anclajes particularmente sociológicos, emergió lo revelador de las relaciones sociales; desde lo extremadamente simple y obvio hasta lo complejo y oculto, marcado siempre por una expresión cultural que escapa a hacer generalizaciones. El capital, el habitus y el campo específico de Enfermería en la comunidad aparecen a partir de una práctica específica, de su historia, de las experiencias que cada una de las Enfermeras les asigna y re-significa a partir de su relación con el pasado, presente y futuro. Aunque también, las estructuras de poder, el papel de los significados rituales, la construcción social se debaten, finalmente, a partir de un medio ambiente y del mismo entorno social y todo esto explica la complejidad de lo social.

Se rescató lo que las Enfermeras dijeron respecto de su actividad profesional, en este caso, la parte invisible, no pragmática ni dogmática de su hacer cotidiano; han mani- 
festado como sentido de su trabajo, la relación de ayuda y la perspectiva existencial que tienen como seres humanos, como parte central de su trabajo. Así los sentimientos, principalmente de ayuda que toma matices de amor, deseo de cuidar, benevolencia, involucramiento, dar, entrega, contacto, confianza, etc. son frecuentemente nombrados dentro de su práctica.

Por ello adquiere relevancia conocer estos valores, las normas y el sentido que adquieren esos espacios sociales concretos, construidos ex profeso para dar cabida a expresiones particulares de las relaciones sociales, que lo mismo corren de lo institucional hasta lo privado, de lo familiar y personal, de la motivación personal al empleo pagado.

Este ejercicio intelectual, ha podido vislumbrar como se movilizan las Enfermeras comunitarias, que les motiva, que les limita; desde la perspectiva de la docencia en Enfermería, se ha abierto un camino lleno de posibilidades para tratar de comprender por qué ocurren así las cosas y por dónde se puede trabajar para ir delineando las fronteras del hacer de la Enfermería, especialmente en el área familiar. Al final de cuentas lo que se busca en la Enfermería en la actualidad es definir el campo disciplinario y este tipo de estudios aportan miradas múltiples a los fenómenos que la Enfermería enfrenta.

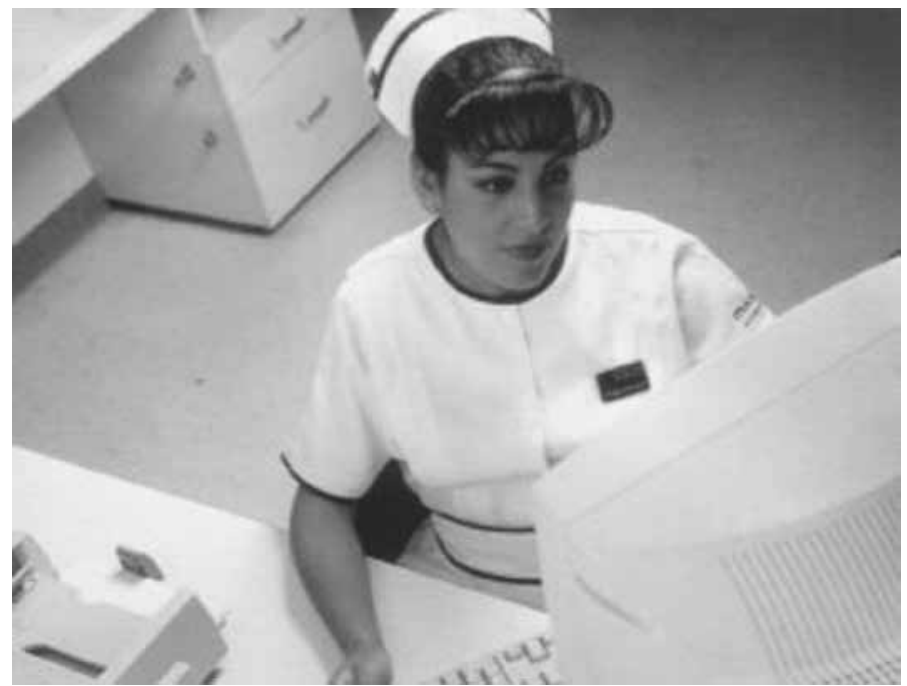

\section{REFERENCIAS BIBLIOGRÁFICAS}

1 Bourdieu P. Estructuras, habitus y prácticas en el sentido práctico. España: Taurus Humanidades; 1991:91-111

2 Bateson, Gregory: Pasos para una ecología de la mente. Carlos Lohlé, Buenos Aires, 1980:449-465

3 De la Cuesta C. Características de la investigación cualitativa y su relación con la Enfermería. Invest Educ Enferm [Publicación periódica en línea] 1997. Sept [citada 2000 enero
15];15(2):[13 pantallas]. Se encuentra en http://tone.udea. edu.co/revista/sep97/caracter.htm.

4 De la Cuesta C. Contribución de la investigación a la práctica clínica de los cuidados de Enfermería. La perspectiva de la investigación cualitativa. Enferm Clínica 1998; 8(4):38-42

5 De la Cuesta C. Investigación cualitativa y práctica de Enfermería. Invest Educ Enferm [Publicación periódica en línea] 1999. Sept [citada 2000 enero 15];17(2):[9 pantallas]. Se encuentra en http://tone.udea.edu.co/revista/sep99/investigacion. htm.

6 Heggenhougen K. Antropología y salud pública: más allá de las medidas cuantitativas. [Sitio en Internet] Cultura, Salud y Reproducción. Disponible en http://www.hsph.harvard. edu/grhf/_Spanish/course/sesion2/antro.htm Acceso el 15 de enero 2003.

7 Mella O. Naturaleza y orientaciones teórico-metodológicas de la investigación cualitativa. [Sitio en Internet] Red Latinoamericana de información y documentación en educación. Disponible en http://www.reduc.cl/reduc/mella.pdf. Acceso el 20 de enero 2002.

8 Nigenda G. y Langer A. Métodos cualitativos para la investigación en salud pública: situación actual y perspectivas. [sitio en Internet] Cultura, Salud y Reproducción. Disponible en http:// www.hsph.harvard.edu/Organizactions/healthnet/frame4/frame4.html. Acceso el 5 de marzo 2001.

9 Valles M. Diseños y estrategias metodologicas en los estudios cualitativos en Técnicas cualitativas de investigación social. Reflexión metodologica y practica profesional. España: Síntesis Sociología; 2000:69-101

10 Glaser B. and Strauss A. El muestreo teórico in The discovery of grounded theory: strategies for qualitative research. Chicago: (trad. Mecanografiada por S. Sanahuja del capitulo III). Aldine de Gruyter; 1967:45

11 Rodríguez G., Gil J. y García E. Selección de informantes y recogida de datos en Metodología de la investigación cualitativa. Colección Biblioteca de Educación. $2^{\mathrm{a}}$ ed. España: Aljibe; 1999.

12 Strauss A. and Corbin J. Basic of qualitative research: grounded theory procedures and thecniques. Newbury Park CA: Sage Publications; 1990:188-193

13 Sanahuja S. y Silva A. Muestreo teórico y estudios del discurso. Una propuesta teórico-metodológica para la generación de categorías significativas en el campo del análisis del discurso. II Coloquio Nacional de Investigadores en Estudio del Discurso. [Sitio en Internet] Sociedad Argentina de Información. Disponible en http://www.sai.com.ar/KUCORIA/discurso. html. Acceso el 23 de agosto 2002.

14 Alonso C, Martín B. Ormaechea S. Obstáculos que afectan la relación teoría-práctica en las prácticas de formación: el caso particular de los practicantes de la carrera de Ciencias de la Educación de la Universidad nacional del Nordeste. [Sitio en 
Internet] Universidad Nacional del Nordeste. Disponible en http://www.unne.edu.ar/cyt/2000/2_humanisticas/h_pdf/ h_020.pdf. Acceso el 17 de septiembre 2002

15 Jociles Rubio M.I. El análisis del discurso: de cómo utilizar desde la antropología social la propuesta analítica de Jesús Ibáñez. Revista del Ateneo de Antropología [publicación periódica en línea]; (0): [1 pantalla]. Se encuentra en http://www.ucm. es/info/dptoants/ateneo/start.html.

16 Valles M. Introducción a la metodología de análisis cualitativo: panorámica de procedimientos y técnicas en Técnicas cualitativas de investigación social. Reflexión metodológica y práctica profesional. España: Síntesis Sociología; 2000:343-350

17 Bryman A. y Burgess R. Introducción a los avances en el análisis de datos cualitativos en Analyzing Qualitative Data. Londres. Traducción para uso interno Universidad Autónoma de Nuevo León. México. 1994

18 Conde F. Procesos e instancias de reducción/formalización de la multidimensionalidad de lo real: procesos de institucionalización/reificación social en la praxis de la investigación social en Métodos y técnicas cualitativas de investigación en ciencias sociales. Compiladores J. M. Delgado y J. Gutiérrez. Síntesis. España. 1994.

19 Almonacid C. El empresariado chileno como actor educativo. [Sitio en Internet] Pontificia. Universidad Católica de Chile. Disponible en http://www.geocities.com/Athens/Delphi/3150/meto.html. Acceso el 5 agosto 2002

20 Navarro P. y Díaz C. Análisis de contenido en Métodos y técnicas cualitativas de investigación en ciencias sociales. Compiladores J. M. Delgado y J. Gutiérrez. Síntesis. España. 1994:177-224.

21 Fernández F. El análisis de contenido como ayuda metodológica para la investigación en Rev Cien Soc Univ Cost Ric 2002;2(96):35-54.

22 Jociles Rubio M.I. El análisis del discurso: de cómo utilizar desde la antropología social la propuesta analítica de Jesús Ibáñez. Revista del Ateneo de Antropología [publicación periódica en línea]; (0): [1 pantalla]. Se encuentra en http://www.ucm. es/info/dptoants/ateneo/start.html.

23 Bryman A. y Burguess R. Introducción a los avances en el análisis de datos cualitativos en Analyzing Qualitative Data. Londres. Traducción para uso interno Universidad Autónoma de Nuevo León. México. 1994

24 Cisneros Puebla ,C.A Computer-assisted qualitative analysis. Sociologias. [publicación periódica en línea] 2003. Jan.-June [25 pantallas]. Se encuentra en http://www.scielo.br/scielo. php?script=sci_arttext\&pid=S1517-45222003000100010\&l

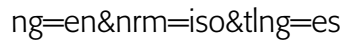

25 Taylor S. y Bogdan R. El trabajo con los datos. Análisis de los datos en la investigación cualitativa en Introducción a los métodos cualitativos de investigación. Barcelona: Paidós; 1993:152-174.
26 Arias M. La triangulación metodológica: sus principios, alcances y limitaciones. Invest Educ Enferm. [publicación periódica en línea] 2000. Marz [citada agosto 2002 02]; 18(1):[26 pantallas]. Se encuentra en http://tone.udea.edu.co/revista/ mar2000/Triangulacion.html

27 Morse J. Approaches to qualitative-quantitative methological triangulation. Nurs Res. 1991; 40(2):120-123

28 Bourdieu P. Estructuras, habitus y prácticas en el sentido práctiCo. España: Taurus Humanidades; 1991:91-111

29 Bourdieu P. El espacio social y el espacio simbólico en Razones prácticas. Sobre la teoría de la acción. Barcelona: Anagrama; 1997:11-32

30 Germaná C. Pierre B. Sociología del poder y violencia simbólica Rev Sociol [publicación periódica en línea] 1999. ; 11(12): [4 pantallas]. Se encuentra en:

http://sisbib.unmsm.edu.pe/bibvirtual/publicaciones/sociolo$\mathrm{gia} / \mathrm{vol} 11$ /art012.htm

31 Giménez, G. La sociología de Pierre Bourdieu en Antología de teoría sociológica contemporánea (coord.), Perspectivas teóricas contemporáneas de las Ciencias Sociales, Facultad de Ciencias Políticas y Sociales. UNAM. México. 1999

32 Montesinos R. y Martínez G. Las rutas elementales de la cultura. Casa del Tiempo [publicación periódica en línea] Dic 2001Ene 2002 [38 pantallas]. Se encuentra en http://www.difusioncultural.uam.mx/revista/dic01 ene02/montesinos.html.

33 Thévenon E. Pierre Bourdieu Una nueva visión del mundo social. Label France. [publicación periódica en línea] julio 2002; 47. Se encuentra en http://www.france.diplomatie. fr/label_france/47/es/20.html

34 Foucault M. Los medios del buen encauzamiento en Vigilar y castigar. México: Siglo XXI Editores; 1999:175-198

35 Alonso C, Martín B. y Ormaechea S. Obstáculos que afectan la relación teoría-práctica en las prácticas de formación: el caso particular de los practicantes de la carrera de Ciencias de la Educación de la Universidad Nacional del Nordeste. [Sitio en Internet] Universidad Nacional del Nordeste. Disponible en http://www.unne.edu.ar/cyt/2000/2_humanisticas/h_pdf/ h_020.pdf. Acceso el 16 de septiembre 2002

36 Benner P. Práctica progresiva en Enfermería: Manual de comportamiento profesional. Colección Enfermería y Sociedad. Barcelona: Grijalbo; 1987:29-40

\section{DIRECCIÓN PARA CORRESPONDENCIA:}

Mtra. Rocío del Carmen Guillén Velasco: roxy40_mx@yahoo.com.mx Mtra. Laura Morán Peña: eneolm@yahoo.com 